\title{
WEIGHTED GROUP ALGEBRA AS AN IDEAL IN ITS SECOND DUAL SPACE
}

\author{
F. GHAHRAMANI
}

\begin{abstract}
For a locally compact group $G$ let $L^{\prime}(G, \omega \lambda)$ be a weighted group algebra. We characterize compact and weakly compact multipliers on $L^{\prime}(G, \omega \lambda)$. This characterization is employed to find a necessary and sufficient condition for $L^{\prime}(G, \omega \lambda)$ to be an ideal in its second dual space, where the second dual is equipped with an Arens product. In the special case where $\omega(t)=1(t \in G)$, we deduce a result due to $\mathrm{K}$. P. Wong that if $G$ is a compact group, then $L^{1}(G, \lambda)$ is an ideal in its second dual space and its converse due to $\mathrm{S}$. Watanabe.
\end{abstract}

1. Introduction. Following the work of W. G. Bade and H. G. Dales [4] on compact elements of $L^{1}\left(R^{+}, \omega\right)$, in [9] we have characterized elements $g \in L^{1}(G, \omega \lambda)$ for which the operator $\rho_{g}: f \mapsto f * g\left(f \in L^{\prime}(G, \omega \lambda)\right)$ is compact, where we have called them compact elements. In this paper we prove that every right (resp. left) compact multiplier $T$ on $L^{1}(G, \omega \lambda)$ is represented by a right (resp. left) compact element $g \in L^{1}(G, \omega \lambda)$, such that $T(f)=f * g\left(f \in L^{1}(G, \omega \lambda)\right.$, and for the left multiplier $T$ we have $T(f)=g * f\left(f \in L^{1}(G, \omega \lambda)\right)$. We also prove that every right (resp. left) weakly compact multiplier is a right (resp. left) compact multiplier.

K. P. Wong in [17] has proved that if $G$ is a compact group and if the second dual space of $L^{\prime}(G, \lambda)$ is equipped with an Arens product, then the canonical image of $L^{\prime}(G, \lambda)$ in its second dual space is an ideal. For the converse of the Wong's result $\mathrm{S}$. Watanabe gave two different proofs [ 15 and $\mathbf{1 6}]$. Other proofs were also provided by M. Grosser [11] and D. L. Johnson [12].

Using our characterization of the weakly compact elements, we find a necessary and sufficient condition for a weighted group algebra to be an ideal in its second dual space, where the second dual is equipped with an Arens product. In particular, we obtain other proofs for the results of Wong and Watanabe.

2. Preliminaries. Throughout, $G$ will be a locally compact group and $\lambda$ a fixed left Haar measure on $G$. A positive and continuous function $\omega$ on $G$ satisfying $\omega(s t) \leqslant$ $\omega(s) \omega(t)(s, t \in G)$ will be called a weight function. For a weight function $\omega$, let $L^{1}(G, \omega \lambda)$ be the Banach space of all complex functions $f$ such that

$$
\|f\|=\int_{G}|f(t)| \omega(t) d \lambda(t)<\infty
$$

Received by the editors March 1, 1983.

1980 Mathematics Subject Classification. Primary 43A20, 43A22; Secondary 47B05.

Key words and phrases. Weighted group algebra, compact multiplier, Arens product. 
where as usual we equate functions $\lambda$ almost everywhere. Under convolution product

$$
(f * g)(x)=\int_{G} f\left(x y^{-1}\right) g(y) d \lambda(y) \quad\left(f, g \in L^{1}(G, \omega \lambda), \lambda \text { a.e. } x \in G\right),
$$

$L^{1}(G, \omega \lambda)$ becomes a Banach algebra. When $\omega(t) \geqslant 1$, the algebra $L^{1}(G, \omega \lambda)$ is called a Beurling algebra [13].

As in [8 and 9] we appeal to a larger algebra of measures; let $M(G, \omega)$ be the Banach space of all complex regular Borel measures $\mu$ on $G$ such that

$$
\|\mu\|=\int_{G} \omega(t) d|\mu|(t)<\infty .
$$

If $C_{0}(G, \omega)$ is the Banach space of all functions $f$ on $G$ such that $f / \omega \in C_{0}(G)$ and $\|f\|=\sup _{x \in G}|f(x) / \omega(x)|$, then by the pairing

$$
\langle\mu, \psi\rangle=\int_{G} \psi(x) d \mu(x) \quad\left(\mu \in M(G, \omega), \psi \in C_{0}(G, \omega)\right),
$$

we have $\left(C_{0}(G, \omega)\right)^{\prime}=M(G, \omega)$, and we can define the product of $\mu, \nu \in M(G, \omega)$ by

$$
\int_{G} \psi(x) d(\mu * \nu)(x)=\int_{G} \int_{G} \psi(x y) d \mu(x) d \nu(y) \quad\left(\psi \in C_{0}(G, \omega)\right) .
$$

Let $X$ be a Banach space and $X^{\prime}$ be the dual of $X$. If $w=\sigma\left(X, X^{\prime}\right)$ is the weak topology on $X$, and $U$ is the open unit ball of $X$, we call an operator $T$ on $X$ weakly compact if the weak closure of $T(U)$ in $X$ is weakly compact. On $M(G, \omega)$ we consider two topologies other than the norm topology and the weak topology: they are (I) the topology (so) in which a net $\left(\mu_{\alpha}\right)$ tends to a measure $\mu$ (so) if $\mu_{\alpha} * f \stackrel{\|\cdot\|}{\rightarrow} \mu * f$, for every $f \in L^{\mathrm{l}}(G, \omega \lambda)$, and (II) the topology

$$
w^{*}=\sigma\left(M(G, \omega), C_{0}(G, \omega)\right) .
$$

The map $f \mapsto \mu_{f}$, where $d \mu_{f}(x)=f(x) d \lambda(x)$, defines an isometric isomorphism from $L^{1}(G, \omega \lambda)$ into $M(G, \omega)$, and $L^{1}(G, \omega \lambda)$ can be identified with a closed ideal of $M(G, \omega)$.

We call an element $g \in L^{1}(G, \omega \lambda)$ right compact (resp. right weakly compact) if the operator $\rho_{g}: f \mapsto f * g$ is compact (resp. weakly compact). We define left (resp. left weakly) compact elements by replacing $f * g$ by $g * f$ in the above definition.

LEMMA 2.1. The algebra $L^{1}(G, \omega \lambda)$ possesses a bounded approximate identity $\left(f_{i}\right)$ such that each $f_{i}$ is a continuous function with compact support and there exists a compact set $S$ containing the supports of all the $f_{i}$.

Proof. It is well known that the algebra $L^{1}(G, \lambda)$ has a bounded approximate identity $\left(f_{i}\right)$ consisting of continuous functions with compact support, where for each $f_{i}$ the support of $f_{i}$ contains the identity element $e$ of $G$ [5, Proposition 69.16]. Moreover, we can assume that there exists a compact set $S$ containing the supports of all the $f_{i}$. The net $\left(f_{i}\right)$ can then, for every weight function $\omega$, serve as a bounded approximate identity for $L^{1}(G, \omega \lambda)$. Indeed, if $g \in L^{1}(G, \omega \lambda)$ is with compact 
support $K$, then since $e \in S$, we have $K \subset K S$, whence,

$$
\begin{aligned}
\left\|g-g * f_{i}\right\|_{\omega} & =\int_{G}\left|g(t)-\left(g * f_{i}\right)(t)\right| \omega(t) d \lambda(t) \\
& \leqslant\left(\sup _{x \in K S} \omega(x)\right) \int_{G}\left|g(t)-\left(g * f_{i}\right)(t)\right| d \lambda(t) \\
& =\left(\sup _{x \in K S} \omega(x)\right)\left\|g-g * f_{i}\right\|_{1} .
\end{aligned}
$$

Likewise,

$$
\left\|g-f_{i} * g\right\|_{\omega} \leqslant\left(\sup _{x \in S K} \omega(x)\right)\left\|g-f_{i} * g\right\|_{1} .
$$

Since the set of functions with compact support is dense in $L^{1}(G, \omega \lambda)$, the lemma is proved.

LEMMA 2.2. Suppose $\left(f_{i}\right)$ is a bounded approximate identity for $L^{1}(g, \omega \lambda)$ as described in Lemma 2.1. Then $f_{i} \rightarrow \delta_{e}$ in the $w^{*}$-topology of $M(G, \omega)$, where $e$ is the identity element of $G$ and $\delta_{e}$ is the unit mass concentrated at $e$.

Proof. First we consider the case $\omega(t)=1(t \in G)$. We have $f_{i} * g \rightarrow g=\delta_{e} * g$, in the norm topology of $L^{1}(G, \lambda)$. Hence $f_{i} \rightarrow \delta_{e}$ in the $w^{*}$-topology of $M(G)[10$, Lemma 1.1.1]. Thus, in this case we have

$$
\int_{G} \psi(x) f_{i}(x) d \lambda(x) \rightarrow \psi(e) \quad\left(\psi \in C_{0}(G)\right) .
$$

For a general $\omega$, let $\varphi \in C_{0}(G, \omega)$. Then

$$
\left\langle\varphi, f_{i}\right\rangle=\int_{G} \varphi(x) f_{i}(x) d \lambda(x) .
$$

Since the $f_{i}$ vanish off the compact set $S$, the function $\varphi$ can be assumed to vanish at infinity. Then, from (1) and (2) it follows $\left\langle\varphi, f_{i}\right\rangle \rightarrow \varphi(e)=\left\langle\varphi, \delta_{e}\right\rangle$, and the lemma is proved.

LEMMA 2.3. Suppose $T$ is a right (resp. left) multiplier on $L^{1}(G, \omega \lambda)$. Then there exists $\mu \in M(G, \omega)$ such that

$$
T(f)=f * \mu \quad\left(f \in L^{1}(G, \omega \lambda)\right),
$$

(resp. $T(f)=\mu * f\left(f \in L^{1}(G, \omega \lambda)\right)$.

Proof. We consider the right multiplier case. Let $\left\{f_{i}: i \in I\right\}$ be a bounded approximate identity for $L^{1}(G, \omega \lambda)$. The set $\left\{T\left(f_{i}\right): i \in I\right\}$ is a bounded subset of $M(G, \omega)$. Thus, by the Banach-Alaoglu theorem, there exists a subnet $\left\{f_{j}: j \in J\right\}$ of $\left\{f_{i}: i \in I\right\}$ and a measure $\mu \in M(G, \omega)$, such that $T\left(f_{j}\right) \rightarrow \mu$ in the $\omega^{*}$-topology of $M(G, \omega)$. Now if $f \in L^{1}(G, \omega \lambda)$, then

$$
T\left(f * f_{j}\right) \stackrel{\|\cdot\|}{\rightarrow} T(f) \text { and } T\left(f * f_{j}\right)=f * T\left(f_{j}\right) \stackrel{w^{*}}{\rightarrow} f * \mu
$$

and the lemma is proved. 


\section{Compact and weakly compact multipliers.}

THEOREM 3.1. The following are equivalent.

(I) $T$ is a weakly compact right multiplier on $L^{1}(G, \omega \lambda)$.

(II) $T$ is a compact right multiplier on $L^{\mathrm{l}}(G, \omega \lambda)$.

(III) There exists $g \in L^{1}(G, \omega \lambda)$ such that $T(f)=f * g\left(f \in L^{1}(G, \omega \lambda)\right)$ and such that the function

$$
x \mapsto \int_{G} \frac{\omega(x y)}{\omega(x)}|g(y)| d \lambda(y) \quad(x \in G)
$$

vanishes at infinity.

Proof. (I) $\Rightarrow$ (II). By Lemma 2.3 there exists $\mu \in M(G, \omega)$ such that $T(f)=f * \mu$ $\left(f \in L^{\prime}(G, \omega \lambda)\right.$ ). First, we show that $\mu \in L^{\mathrm{l}}(G, \omega \lambda)$. Let $\left\{f_{i}: i \in I\right\}$ be a bounded approximate identity for $L^{\prime}(G, \omega \lambda)$, same as in Lemma 2.1. By weak compactness of $T$ there exist a subnet $\left(f_{j}\right)$ of $\left(f_{i}\right)$ and $g \in L^{1}(G, \omega \lambda)$ such that $T\left(f_{j}\right)=f_{j} * \mu \rightarrow g$ in the weak topology of $L^{1}(G, \omega \lambda)$. By Lemma $2.2, f_{j} * \mu \stackrel{\omega^{*}}{\rightarrow} \delta_{0} * \mu=\mu$. Hence $\mu=g \in$ $L^{1}(G, \omega \lambda)$.

Next, writing $g$ instead of $\mu$, we first prove that for every $i \in I$, the operator $h \mapsto h * g * f_{i}\left(h \in L^{1}(G, \omega \lambda)\right)$ is a compact operator [4, Theorem 2.9].

Let $\left(k_{n}\right)$ be a bounded sequence in $L^{1}(G, \omega \lambda)$. Since $h \mapsto h * g$ is weakly compact, there is a subsequence $\left(k_{n}\right)$ of $\left(k_{n}\right)$ and an element $k_{0}$ of $L^{\prime}(G, \omega \lambda)$ with $k_{n_{1}} * g \rightarrow k_{0}$ weakly. Hence, to show that $h \mapsto h * g * f_{i}$ is compact, it suffices to show that, if $h_{n} \rightarrow h_{0}$ weakly in $L^{1}(G, \omega \lambda)$, then $h_{n} * f_{i} \rightarrow h_{0} * f_{i}$ in the norm topology of $L^{1}(G, \omega \lambda)$. Suppose then that $h_{n} \rightarrow h_{0}$ weakly. For each $x \in G$,

$$
\left[f_{i} *\left(h_{n}-h_{0}\right)\right](x)=\int_{G} f_{i}\left(x y^{-1}\right)\left[h_{n}(y)-h_{0}(y)\right] d \lambda(y) .
$$

If $\varphi(y)=f_{i}\left(x y^{-1}\right)(y \in G)$, then $\varphi$ is bounded and has compact support, and so $\sup \{|\varphi(y) / \omega(y)|: y \in G\}<\infty$. Thus, $\varphi \in L^{\infty}(G, \omega \lambda)$, and so $\left(h_{n} * f_{i}\right)(x) \rightarrow$ $\left(h_{0} * f_{i}\right)(x)$, as $n \rightarrow \infty$. By [7, III, 6.13], $h_{n} * f_{i} \rightarrow h_{0} * f_{i}$, as $n \rightarrow \infty$, in measure on subsets of $G$ of finite measure (for the measure $\omega(t) d \lambda(t)$ ). But $h_{n} * f_{i} \rightarrow h_{0} * f_{i}$ weakly as $n \rightarrow \infty$, and so by [7, IV, 8.12], $h_{n} * f_{i} \rightarrow h_{0} * f_{i}$ in $L^{\prime}(G, \omega \lambda)$, as $n \rightarrow \infty$. Now, we have $g=\lim g * f_{i}$ in $L^{1}(G, \omega \lambda)$, and the compactness of $T$ follows.

Obviously (II) $\Rightarrow$ (I). Thus, (I) and (II) are equivalent.

To prove the equivalence of (II) and (III), we note that if $T$ is a compact multiplier on $L^{1}(G, \omega \lambda)$, then by an argument similar to the one in the first paragraph there is a $g \in L^{1}(G, \omega \lambda)$ such that $T(f)=f * g\left(f \in L^{1}(G, \omega \lambda)\right)$. Then, the equivalence of (II) and (III) follows from [9, Theorem 1].

A similar result holds for left multipliers.

COROllary 3.2 (SAKaI [14, TheOREM 1]). If $G$ is a locally compact noncompact group, then 0 is the only right (resp. left) weakly compact element of $L^{1}(G, \lambda)$.

Proof. This follows from Theorem 3.1 and [9, Theorem 1]. 
4. $L^{1}(G, \omega \lambda)$ as an ideal. Suppose $A$ is a Banach algebra, then the second dual space $A^{\prime \prime}$ of $A$ may be equipped with two Banach algebra products, known as first (or left) and second (or right) Arens products [2, 3 and 6] each of which is an extension of the original product in $A$ as canonically embedded in $A^{\prime \prime}$. We assume $A^{\prime \prime}$ is equipped with the left Arens product. For each $a \in A$, we let $\pi(a)$ denote the canonical image of $a$ in $A^{\prime \prime}$, and $\rho_{a}$ and $\lambda_{a}$ be the operators $\rho_{a}: b \mapsto b a(b \in A)$ and $\lambda_{a}: b \mapsto a b(b \in A)$.

Below we give a different proof to a result due to Watanabe [15].

Proposition 4.1. Let $A$ be a Banach algebra. Then the following statements are equivalent.

(I) For each $a \in A, \rho_{a}$ (resp. $\lambda_{a}$ ) is right (resp. left) weakly compact on $A$.

(II) $\pi(A)$ is a right (resp. left) ideal of $A^{\prime \prime}$.

Proof. For every $a \in A$ the operator $\rho_{a}$ is weakly compact if and only if $\left(\rho_{a}\right)^{* *} A^{\prime \prime} \subset \pi(A)$, where $\rho_{a}^{* *}$ is the second adjoint of $\rho_{a}[7, \mathrm{VI}, 4.2]$. But $\rho_{a}^{* *}$ is the same as multiplication on the right by $\pi(a)$ in $A^{\prime \prime}$. Hence for every $a \in A, \rho_{a}$ is weakly compact if and only if $A^{\prime \prime} \pi(a) \subset \pi(A)$. The corresponding result for $\lambda_{a}$ can, similarly, be proved and the equivalence of (I) and (II) is established.

COROllary 4.2. A necessary and sufficient condition for the canonical image of $L^{\prime}(G, \omega \lambda)$ in its second dual to be a right (resp. left) ideal is that for every $g \in L^{1}(G, \omega \lambda)$, the function

$$
x \mapsto \int_{G} \frac{\omega(x y)}{\omega(x)}|g(y)| d \lambda(y) \quad(x \in G),
$$

(resp. $x \mapsto \int_{G} \omega(y x) / \omega(x)|g(y)| d \lambda(y)(x \in G)$ ) vanishes at infinity.

Proof. This follows from Theorem 3.2 and Proposition 4.1.

COROLlARY 4.3. If $G$ is a compact group, then the canonical image of $L^{1}(G, \omega \lambda)$ in its second dual is an ideal.

Proof. For every $g \in L^{1}(G, \omega \lambda), \rho_{g}$ and $\lambda_{g}$ are compact [9, Corollary 1], and hence weakly compact.

If in the above corollary we let $\omega(t)=1(t \in G)$, then we obtain Wong's result.

Corollary 4.4 (S. Watanabe $[15,16])$. If $L^{1}(G, \lambda)$ is a right (resp. left) ideal in its second dual space, then $G$ is compact.

Proof. If $L^{1}(G, \lambda)$ is a right (resp. left) ideal in its second dual space, then by Proposition 4.1 , for every $g \in L^{1}(G, \lambda), \rho_{g}$ (resp. $\lambda_{g}$ ) is weakly compact, and the result follows from Corollary 3.2.

REMARK 4.5. We have seen that for the case $\omega(t)=1(t \in G)$, if $0 \neq g \in L^{1}(G, \lambda)$, then $\rho_{g}$ is compact if and only if $\lambda_{g}$ is compact (in which case $G$ is a compact group). On the other hand, if for some $0 \neq g \in L^{1}(G, \lambda), \rho_{g}$ is compact, then this holds for every element of $L^{1}(G, \lambda)$. These considerations amount to the following question.

Question 1. Is every right (resp. left) compact element of $L^{1}(G, \omega \lambda)$ a left (resp. right) compact element? 
Question 2. Does from right (resp. left) compactness of one nonzero element follow that all the other elements of $L^{1}(G, \omega \lambda)$ are right (resp. left) compact elements?

ACKnowledgments. The author wishes to thank Dr. H. G. Dales for a preprint of [4].

\section{REFERENCES}

1. C. Akemann, Some mapping properties of the group algebras of a compact group, Pacific J. Math. 22 (1967), 1-8.

2. R. Arens, Operators in function classes, Monatsh. Math. 55 (1951), 1-19.

3. The adjoint of a bilinear operation, Proc. Amer. Math. Soc. 2 (1951), 839-848.

4. W. G. Bade and H. G. Dales, Norms and ideals in radical convolution algebras, J. Funct. Anal. 41 (1981), 77-109.

5. S. K. Berberian, Lectures in functional analysis and operator theory, Springer-Verlag, Berlin and New York, 1974.

6. J. Duncan and S. A. R. Hosseiniun, The second dual of a Banach algebra, Proc. Roy. Soc. Edinburgh Sect. A. 84 (1979), 309-325.

7. N. Dunford and J. Schwartz, Linear operators, Part 1, Interscience, New York, 1958.

8. F. Ghahramani, Homomorphisms and derivations on weighted convolution algebras, J. London Math. Soc. 21 (1980), 149-161.

9. Compact elements of weighted group algebras, Pacific J. Math. (to appear).

10. F. P. Greenleaf, Norm decreasing homomorphisms of group algebras, Pacific J. Math. 15 (1965), $1187-1219$.

11. M. Grosser, $L^{\prime}(G)$ as an ideal in its second dual space, Proc. Amer. Math. Soc. 73 (1979), 363-364.

12. D. L. Johnson, A characterization of compact groups, Proc. Amer. Math. Soc. 74 (1979), 381-382.

13. H. Ricter, Classical harmonic analysis and locally compact groups, Oxford Univ. Press, Oxford, 1968.

14. S. Sakai, Weakly compact operators on operator algebras, Pacific J. Math. 14 (1964), 659-664.

15. S. Watanabe, A Banach algebra which is an ideal in the second dual space, Sci. Rep. Niigata Univ. Ser. A 11 (1974), 95-101.

16. A Banach algebra which is an ideal in the second dual space II, Sci. Rep. Niigata Univ. Ser. A 13 (1976), 43-48.

17. K. P. Wong, On the Arens product and annihilator algebras, Proc. Amer. Math. Soc. 30 (1971), 79-83.

Department of Mathematics, University for Teacher Education, 49, Mofateh Avenue, Tehran, IRAN 